This is the final peer-reviewed accepted manuscript of:

Mendolicchio, C., Pietra, T. Full and constrained Pareto efficiency with incomplete financial markets. Econ Theory 71, 211-234 (2021). https://doi.org/10.1007/s00199-019-01239-y

The final published version is available online at: https://doi.org/10.1007/s00199019-01239-y

Rights / License:

The terms and conditions for the reuse of this version of the manuscript are specified in the publishing policy. For all terms of use and more information see the publisher's website. 


\title{
Full and Constrained Pareto Efficiency with Incomplete Financial Markets
}

\author{
C. Mendolicchio ${ }^{1}$, T. Pietra ${ }^{2 \star}$ \\ 1 Department of Economics, Università di Genova, Genova, Italy, e-mail: concetta.mendolicchio@economia.unige.it \\ 2 Department of Statistics, Università di Bologna, Italy, e-mail: tito.pietra@unibo .it
}

The date of receipt and acceptance will be inserted by the editor

\begin{abstract}
We study the efficiency properties of equilibria in general equilibrium economies with incomplete financial markets. We focus the analysis on economies with a finitely large number of agents and initial endowments close to a Pareto optimal one. Consider an economy with a Pareto optimal equilibrium allocation and a sufficiently small, but non-zero, amount of trade. If the matrix of the derivatives of the indirect utility functions with respect to prices has maximal rank (equal to the number of non-numeraire commodities), then locally all the economies have a unique, constrained Pareto efficient equilibrium. To the contrary, pick a Pareto optimal initial endowment. Then, locally, there are open sets of economies with a unique, constrained Pareto efficient equilibrium and other open sets of economies with a unique constrained Pareto inefficient equilibrium. The key step of the analysis is based on the observation that, in some small open neighborhood of an economy with a Pareto optimal initial endowment, we can partially characterize constrained Pareto optimal equilibria as the optimal solution of a well-defined, strictly-concave optimization problem.
\end{abstract}

Keywords: GEI, constrained Pareto efficiency, numeraire assets JEL classification: D51, D52

\footnotetext{
* Corresponding author. This author acknowledges the financial support of MIUR-PRIN 2015. We are grateful to two anonymous referees for helpful comments and suggestions. The responsibility for any remain error or obscurity is, as usual, just our own.
} 


\section{Introduction}

Our purpose is to study the efficiency properties of equilibria when financial markets are incomplete. We adopt the canonical notion of constrained Pareto (CP) efficiency introduced in the GEI literature by [2]: ${ }^{1}$ an equilibrium is $\mathrm{CP}$ efficient if it is not Pareto inferior to any allocation obtained perturbing portfolios and adjusting spot prices to restore market-clearing for all the commodities.

[1-2] have shown that equilibria are generically CP inefficient when the number of agents is small in comparison with the one of non-numeraire commodities. Constrained and - a fortiori - full Pareto efficiency of equilibria may occur, but a generic perturbation of the parameters of the economy suffices to restore CP inefficiency. The logic of their argument is fairly transparent. Starting with an equilibrium, a small portfolio perturbation has no direct effect on agents' welfare. However, since it induces an adjustment of equilibrium commodity prices, it has non-trivial second order welfare effects. Under some restrictions, generically satisfied at each equilibrium, appropriate, feasible portfolio perturbations may induce each profile of commodity price adjustments. Let's consider a particular good. Then, for instance, a price increase makes net sellers better off and net buyers worst off. When markets are complete, the vectors of normalized marginal utilities of incomes at the different spots are identical and utility gains and losses due to a price change must cancel out when aggregating across agents. Therefore, a pure reallocation of income across spots, which is the actual effect of a portfolio redistribution, cannot lead to a Pareto improvement. In GEI, agents' marginal utilities of incomes at the various spots are different. Agents evaluate in different ways gains and losses taking place at different spots and, therefore, a portfolio perturbation may be Pareto improving. This is the basic intuition behind the classical constrained inefficiency result in GEI. For this result to be true, we need three properties: the number of agents must be smaller than the number of non-numeraire commodities; normalized marginal utilities of spot incomes must vary sufficiently across consumers; appropriate portfolio perturbations must allow for each direction of equilibrium commodity price adjustments. The last two properties are generically satisfied at each equilibrium. Hence, equilibria typically fail to be CP efficient when the number of agents is sufficiently small. Notice that the analysis can be carried out at a purely local level, since GEI equilibria are typically dominated by the equilibrium allocations associated with portfolios arbitrarily close to the equilibrium ones. A weakness of this result is that the restriction on the number of agents is far from innocuous, since it is somewhat in contradiction with the assumption of competitive behavior.

A purpose of this paper is to further extend the analysis of CP efficiency in GEI when the number of agents is large, but finite. Unfortunately, for this case, neither CP efficiency nor its lack are generic properties (see [5]).

\footnotetext{
${ }^{1}$ See also [6].
} 
The best we can hope for is to be able to find sufficient conditions for CP efficiency (respectively, inefficiency) characterizing some classes of economies. This is what we accomplish here.

As already mentioned, the canonical approach to the study of the constrained efficiency properties in GEI looks at the welfare effect of small portfolio perturbations. This approach may work to prove the existence of economies with $\mathrm{CP}$ inefficient equilibria, but it does not help to establish $\mathrm{CP}$ efficiency. This last one is a global property so that we must take into account all the equilibrium allocations associated with each feasible portfolio reallocation: it is doubtful that we can obtain any interesting, general result. There are, however, some classes of economies which allow us to partially sidestep this problem because, if a Pareto dominant equilibrium allocation associated with some feasible portfolio exists, it must be arbitrarily close to the equilibrium one, so that a local analysis may suffice. For instance, this happens for the economies sufficiently close to the ones with identical, homothetic state-preference. [5] reports some results for this case. Here, we consider, instead, economies in open neighborhoods of the ones with a fully Pareto optimal equilibrium. In GEI models, this set is negligible (i.e., closed and nowhere dense), but it is still of interest for both technical and substantive reasons, as we will argue below. Moreover, we will consider open sets of economies, close - or containing - the one with a PO equilibrium, so that our results apply to non-negligible sets.

We provide two results. Consider an economy with a unique, Pareto optimal equilibrium. If the matrix of the derivatives of the indirect utility functions with respect to spot prices has full row rank (which is generically true at each equilibrium) and the vectors of excess demand are sufficiently small and dispersed across consumers, then all the economies in some open neighborhood of this exceptional one have a unique, $\mathrm{CP}$ efficient equilibrium. On the other hand, if the reference economy has a no-trade, PO equilibrium, each one of its open neighborhoods contains both open sets of economies with a unique $\mathrm{CP}$ efficient equilibrium and other open sets with a unique $\mathrm{CP}$ inefficient equilibrium. The set of economies with a no-trade, $\mathrm{PO}$ equilibrium is lower dimensional in economy space. However, once again, all the sets we construct are open, so that the two sets of economies, with and without $\mathrm{CP}$ efficient equilibria, have full dimension. ${ }^{2}$

To our mind, the existence of open sets of both CP efficient and inefficient equilibria in each open neighborhood of a no-trade, $\mathrm{PO}$ equilibrium is quite counterintuitive. Economies with PO initial endowments are the canonical example of well-behaved economies, in terms of regularity and comparative statics. However, in GEI models, they are critical with respect to their efficiency properties, because they are the intersection, in parameter space, of paths of economies with $\mathrm{CP}$ efficient equilibria and of other paths with $\mathrm{CP}$ inefficient equilibria. This will be made clear by the examples concluding

${ }^{2}$ The space of economies is parameterized by endowments and utility functions. Therefore, there is no canonical measure theoretic notion of size. 
the paper. The intuitive explanation is that, at no-trade equilibria, there is a collapse of the rank of the matrix describing the derivatives of the indirect utility functions with respect to prices: with no-trade, this matrix is nil. Arbitrarily small changes of the parameters - i.e., of endowments and utility functions - generate matrices spanning completely different subspaces. Some perturbations of the economy allow for the existence of a Pareto improving price change, others do not. For economies close to the reference economy with a no-trade, PO equilibrium, this is enough to imply, respectively, CP efficiency or inefficiency of equilibria.

Constrained optimality of equilibria of all the economies close to one with a PO allocation can appear to be a somewhat more intuitive property, but it is far from obvious in GEI. In fact, we show that it is true only under some other additional restrictions. Our proof rests on the property that a linear welfare function, with appropriate weights, is strictly-concave in portfolios in some open neighborhood of a no-trade PO equilibrium, even when we take into account the induced adjustments of spot prices. This property holds independently of any substantive restriction on the utility functions, but their strict-concavity. To the best of our knowledge, this has never previously been pointed out in the literature.

In view of unpublished results in [3-4] on CP efficiency of equilibria in large economies with well-dispersed characteristics, ${ }^{3}$ it is worthwhile to remark that, in our set-up, the existence of open sets of economies with CP inefficient equilibria holds for all finite economies, without any upper bound on the number of agents. Moreover, both CP efficiency and inefficiency hold for open sets of economies constructed without any restriction on the degree of heterogeneity across agents. To the contrary, in our companion paper, we were able to show existence of CP efficient equilibria just for open sets of economies in a neighborhood of an economy with identical homothetic preferences. Implicitly, this imposed an upper bound on the degree of heterogeneity.

A final remark: we carry out our analysis using as building blocks the indirect utility functions. Under regularity of equilibria, this allows us to take into account the welfare effects of price adjustments in a simple and direct way. Our approach turns out to be quite convenient in dealing with GEI. More generally, it appears to be quite useful when dealing with the welfare analysis of economies with any sort of pecuniary externality.

In the next section, we briefly describe the canonical GEI model and our notation. The main results are reported in Section 3, where we also propose an extended example constructed for economies in a neighborhood of an economy with a PO endowment.

\footnotetext{
3 These results are mentioned in [1].
} 


\section{The Model}

The economy is a standard two periods GEI model with numeraire assets. There is a finite set of agents $(h=1, \ldots, H)$, and a finite set of commodities $(c=1, \ldots, C)$ at each spot, denoted by $s=0, \ldots, S$. Spot $s=0$ is today, $s>0$ is a state of the world in the next period. A consumption plan is $x_{h} \equiv$ $\left(x_{h}^{0}, x_{h}^{1}, \ldots, x_{h}^{S}\right) \in \mathbb{R}_{+}^{(S+1) C}$. Commodity prices are $p \equiv\left(p^{0}, p^{1}, \ldots, p^{S}\right) \in$ $\mathbb{R}_{++}^{(S+1) C}$ and we normalize the price of good 1 at each spot. There are $J<S$ assets. A portfolio is $b_{h} \equiv\left(b_{h}^{1}, \ldots, b_{h}^{J}\right) \in \mathbb{R}^{J}$. Asset prices are $q \equiv$ $\left(q^{1}, \ldots, q^{J}\right) \in \mathbb{R}^{J}$. Assets' payoffs are defined in terms of the numeraire commodities and described by a full rank, $(S \times J)$ matrix $R$ with rows in general position,

$$
R \equiv\left[\begin{array}{ccc}
r^{11} & & r^{1 J} \\
\vdots & \ddots & \vdots \\
r^{S 1} & & r^{S J}
\end{array}\right]
$$

$Y(q) \equiv\left[-q^{T}, R^{T}\right]^{T}=\left[y^{0}(q), \ldots, y^{S}\right]^{T}$ is the $((S+1) \times J)$ assets' pricepayoffs matrix.

Agent $h$ 's utility function is $u_{h}\left(x_{h}\right)$ and it satisfies the following assumption:

Assumption $U$ : For each $h, u_{h}\left(x_{h}\right)$ is strictly monotone, $C^{2}$, with $D_{x_{h}}^{2} u_{h}\left(x_{h}\right)$ negative-definite, and satisfies the boundary conditions: for each $\bar{x}_{h} \gg 0$, the closure of the set $\left\{x_{h}: u_{h}\left(x_{h}\right) \geq u_{h}\left(\bar{x}_{h}\right)\right\}$ is contained in $\mathbb{R}_{++}^{(S+1) C}$.

Some of our proofs exploit strict-concavity of the utility functions. This restriction is not essential. Intuitively, our results would go through also for differentiably strictly quasi-concave functions. However, they refer to open sets of economies. Since the set of $C^{2}$, strictly-concave functions is open, there is no substantial loss of generality in imposing the stronger restriction, which streamlines somewhat our proofs.

For each $h$, the initial endowment vector is $\omega_{h} \equiv\left(\omega_{h}^{0}, \omega_{h}^{1}, \ldots, \omega_{h}^{S}\right) \in$ $\mathbb{R}_{++}^{(S+1) C}$. Consumers' behavior is described as the optimal solution to the problem: Given $(p, q)$, choose

$$
\left(x_{h}, b_{h}\right) \in \arg \max u_{h}\left(x_{h}\right) \quad \text { subject to } p^{s}\left(x_{h}^{s}-\omega_{h}^{s}\right)=y^{s}(q) b_{h}, s=0, \ldots, S .
$$

Let $\lambda_{h} \in \mathbb{R}_{++}^{S+1}$ be the vector of Lagrange multipliers associated with the optimal solution to problem $(1), V_{h}(p, q)$ be agent $h^{\prime}$ s indirect utility function, and $\tilde{V}_{h}\left(p, q ; \tilde{b}_{h}\right)$ be the $\tilde{b}_{h}$-conditional indirect utility function, which associates the maximum attainable level of utility with prices $(p, q)$ and an exogenously given portfolio $\tilde{b}_{h}$.

We use " $\sim$ " to denote functions and variables referred to the $\tilde{b}$-conditional economy, and the superscript " $T$ " to denote column vectors. Finally, our notation will specify that the demand functions depend upon $(\omega, u)$ just when required to avoid possible misunderstandings. 
Definition 1 An equilibrium is a price vector $(\bar{p}, \bar{q})$ with associated allocation and portfolio profile $\left\{\ldots,\left(\bar{x}_{h}, \bar{b}_{h}\right), \ldots\right\}$ such that:

a. for each $h,\left(\bar{x}_{h}, \bar{b}_{h}\right)$ solves problem (1) given $(\bar{p}, \bar{q})$,

b. $\sum_{h}\left(\bar{x}_{h}-\omega_{h}\right)=0$ and $\sum_{h} \bar{b}_{h}=0$.

Given an equilibrium $(\bar{p}, \bar{q})$ and a portfolio $\tilde{b}$ with $\sum_{h} \tilde{b}_{h}=0$, a $\tilde{b}$-conditional equilibrium is a price vector $(\tilde{p}, \bar{q})$ with allocation $\tilde{x}$ such that:

c. for each $h, \tilde{x}_{h}$ solves problem (1) given $(\tilde{p}, \bar{q})$ and $\tilde{b}_{h}$,

d. $\sum_{h}\left(\tilde{x}_{h}-\omega_{h}\right)=0$.

As standard when testing for the existence of a Pareto superior $\tilde{b}$-conditional equilibrium, we keep fixed the vector of asset prices at their initial equilibrium level and, of course, we just consider feasible portfolio reallocations.

We parameterize economies in terms of endowments and utility functions, and identify their space with $\mathcal{E} \equiv \mathbb{R}_{++}^{(S+1) C H} \times \mathcal{U}$. An economy is $(\omega, u) \in \mathcal{E}$, where $\mathbb{R}_{++}^{(S+1) C H}$ is endowed with the standard topology, $\mathcal{U}$ with the $C^{2}$, compact-open topology, and $\mathcal{E}$ with the product topology, so that $\mathcal{E}$ is a metric space. Our results necessarily require perturbations of the utility functions. Therefore, a set of economies is generic if and only if it is an open and dense subset of $\mathcal{E}$.

By the appropriate version of Walras' law, we can ignore the market clearing conditions for commodity 1 at each spot. Hence, an equilibrium is a zero of the system of the remaining $((S+1)(C-1)+J)$ market clearing equations. We use $z_{h}=\left(z_{h}^{0}, \ldots, z_{h}^{S}\right) \in \mathbb{R}^{(S+1)(C-1)}$ to denote the vector of the excess demand for the non-numeraire commodities.

The notion of CP efficiency, the same of [2], is reported in Definition 2.

Definition 2 An equilibrium $(\bar{p}, \bar{q})$ is constrained Pareto $(C P)$ efficient (or optimal) if there is no profile $\tilde{b} \equiv\left\{\ldots, \tilde{b}_{h}, \ldots\right\}$ with $\sum_{h} \tilde{b}_{h}=0$ such that the associated $\tilde{b}$-conditional equilibrium $\tilde{p}$ satisfies $\tilde{V}_{h}\left(\tilde{p}, \bar{q} ; \tilde{b}_{h}\right) \geq V_{h}(\bar{p}, \bar{q})$, for each $h$, with at least one strict inequality.

\section{Main Results}

We now consider the nexus between economies with a Pareto optimal equilibrium allocation and economies with $\mathrm{CP}$ efficient, and inefficient, equilibria. As well-known, under market incompleteness, full Pareto efficiency of equilibria holds for a negligible set of economies. What is of interest here are the constrained efficiency properties of open sets of economies arbitrarily close to this set. As established in [1-2], CP inefficiency is a generic property when the number of agents is sufficiently small, i.e., when $H \leq(S+1)(C-1)$. In this paper, we are going to show two main results on the properties of economies with a number of agents violating this upper bound. First, we provide conditions such that Pareto optimality of the equilibrium allocation of an economy implies CP efficiency of the equilibria of all the economies 
closed by. This result holds under two restrictions on the equilibrium realization of the excess demand vectors and of the Lagrange multipliers: a) the matrix of the derivatives of the indirect utility functions with respect to commodity prices must have maximal rank $(S+1)(C-1)$. This property is satisfied at all the equilibria of a generic set of economies; ${ }^{4}$ b) the amount of trade at the equilibrium must be sufficiently small. This is always true for some open subset of regular economies: simply take a neighborhood of the economies with a PO initial endowment. ${ }^{5}$ The second result is that, when the $\mathrm{PO}$ allocation is no-trade, there is, instead, no unambiguous implication for the $\mathrm{CP}$ optimality of the equilibria of the neighboring economies: each economy with a no-trade, $\mathrm{PO}$ equilibrium is surrounded by open sets of $\mathrm{CP}$ efficient economies and by other open sets of $\mathrm{CP}$ inefficient ones. A collateral benefit of our argument is that it provides a simple recipe to construct parametric examples of economies with $\mathrm{CP}$ optimal equilibria and with $\mathrm{CP}$ inefficient ones.

With a finite, but large, number of agents, one needs to tackle a basic difficulty: CP efficiency is a statement on the properties of the entire set of $\tilde{b}$-conditional equilibria. Hence, we face a fundamental asymmetry. If an equilibrium allocation is Pareto dominated by a $\tilde{b}$-conditional equilibrium allocation arbitrarily close, evidently it is CP inefficient. Hence, as in the case with "few" agents, a purely local analysis may suffice to show CP suboptimality. To the contrary, to establish CP optimality, we need to consider all the $\tilde{b}$-conditional equilibrium allocations, including the ones bounded away from the equilibrium one. In general, a local analysis is not sufficient anymore. However, we can sidestep this difficulty by restricting the analysis to open sets of economies such that, if the equilibrium is CP suboptimal, it is dominated by some $\tilde{b}$-conditional equilibrium allocation arbitrarily close to the equilibrium one. This is the case, among others, for some open set of GEI economies containing the ones with a unique, Pareto optimal equilibrium, as established in our Lemma 2. For these economies, a local analysis is in fact sufficient to show CP optimality. This simplifies a lot our task.

Even when we can limit ourselves to a local analysis, we face a second difficulty, related to the welfare effects of the price adjustments induced by the exogenously given portfolio perturbations. They are essential, since they are the cause for the possible CP inefficiency, but they make hard to find a simple characterization of $\mathrm{CP}$ optimal equilibria, even locally. This second difficulty can be side-stepped for, at least, two reasonably general classes of economies. If state-preferences are sufficiently close to be homothetic and

4 This property can never be satisfied at a PO equilibrium allocation of an economy $(\bar{\omega}, \bar{u})$. However, it is satisfied at the equilibrium of all the economies in a full dimensional subset of any open neighborhood of $(\bar{\omega}, \bar{u})$. This is what we exploit in our proofs.

5 This is the most obvious example. More generally, both restrictions are satisfied for economies in a full dimensional subset of an open neighborhood of economies $(\widehat{\omega}, \widehat{u})$ lying on the equilibrium fiber of an economy $(\bar{\omega}, \bar{u})$ with a PO endowment, if $\|(\widehat{\omega}, \widehat{u})-(\bar{\omega}, \bar{u})\|$ is sufficiently small. 
identical across consumers, the commodity price adjustments induced by a feasible portfolio perturbation are suitably small. As it turns out, CP efficient equilibria can be written as the optimal solution to a well-defined, strictly-concave planning problem. This is the approach exploited in [5]. Here, we study a second class of economies where, as established in Lemma 4, each equilibrium allocation solving the first order conditions of a welldefined, and strictly-concave, optimization problem is $\mathrm{CP}$ efficient: they are the ones with equilibria sufficiently close to a no-trade $\mathrm{PO}$ allocation.

Let us start with an informal discussion of our strategy of proof. First, remember that, by the generalization of Roy's Lemma to sequential economies, $\frac{\partial V_{h}}{\partial p^{s c}}=-\lambda_{h}^{s} z_{h}^{s c}$. Let $\Lambda\left(\lambda^{n}, z\right)$ be the, normalized, induced $((S+1)(C-1) \times H)$ matrix, for an economy $(\bar{\omega}, \bar{u})$ :

$$
\Lambda\left(\bar{\lambda}^{n}, \bar{z}\right) \equiv\left[\begin{array}{ccc}
-\bar{z}_{1}^{02} & \cdots & -\bar{z}_{H}^{02} \\
\vdots & \ddots & \vdots \\
-\frac{\bar{\lambda}_{1}^{S}}{\lambda_{1}^{0}} \bar{z}_{1}^{S C} & \cdots & -\frac{\bar{\lambda}_{H}^{S}}{\lambda_{H}^{O}} \bar{z}_{H}^{S C}
\end{array}\right] .
$$

This matrix plays a key role in our discussion since it delivers the impacts on individual utilities of the changes of equilibrium prices induced by an exogenous perturbations of the portfolios. Two properties of $\Lambda\left(\bar{\lambda}^{n}, \bar{z}\right)$ must be kept in mind: as observed above, at each PO equilibrium, $\Lambda\left(\bar{\lambda}^{n}, \bar{z}\right)[1]^{T}=0$, because the coefficients $\frac{\bar{\lambda}_{h}^{s}}{\bar{\lambda}_{h}^{o}}$ are agent-invariant and markets clear. Moreover, at each no-trade equilibrium, $\Lambda\left(\bar{\lambda}^{n}, \bar{z}\right)$ is trivial, and its span can be modified in completely different directions by choosing appropriate endowment perturbations which do not affect any equilibrium variables but the vector $z$.

Our first main result is CP optimality of the equilibrium of each economy in some small open neighborhood of an economy with a unique PO equilibrium allocation where the matrix of individual excess demand has maximal rank $(S+1)(C-1)$ and the amount of trade is sufficiently small. Its proof is structured in three steps and exploits heavily the special properties of economies with a PO endowment. Our first observation is formalized in Lemma 2: for each economy sufficiently close to an economy $(\bar{\omega}, \bar{u})$ with a PO equilibrium, each Pareto dominant portfolio, if any exists, must be arbitrarily close to the one of $(\bar{\omega}, \bar{u})$. Hence, in an intuitive sense, for these economies, local CP efficiency entails global CP efficiency. This first property is unrelated to the volume of trade at the equilibrium of $(\bar{\omega}, \bar{u})$. Hence, locally, it holds for each economy with an equilibrium allocation sufficiently close to Pareto optimality. The second step (in Lemma 3) is to notice that an equilibrium which maximizes a welfare function obtained as an appropriately weighted sum of the individual indirect utility functions (evaluated taking into account commodity price adjustments) is CP efficient. This property is fairly obvious. Less obvious is the third result, reported in Lemma 4: in a neighborhood of a PO, no-trade equilibrium, this welfare function is strictly-concave when we use the vector of welfare weights $\left[\ldots, \frac{1}{\lambda_{h}^{0}}, \ldots\right]$. 
Hence, we can characterize its local maxima using the first order conditions of a well-defined, strictly-concave optimization problem. This result provides, locally, a partial characterization of $\mathrm{CP}$ efficient equilibria and allows for a straightforward check of CP optimality. To establish that there is an open set of economies with a $\mathrm{CP}$ efficient equilibrium it suffices to restrict the analysis to economies sufficiently close to one with a no-trade, PO equilibrium (so that Lemma 2 holds) and such that, at their equilibrium, the FOCs are satisfied. Here, there is a final difficulty, crucially related to the properties of the matrix $\Lambda\left(\bar{\lambda}^{n}, \bar{z}\right)$. If we pick as reference an economy $(\bar{\omega}, \bar{u})$ with a PO equilibrium and some sufficiently small, but not trivial, level of trade, the result of Lemma 4 holds and, for all the economies in some open neighborhood of $(\bar{\omega}, \bar{u})$, the FOCs are satisfied at the equilibrium. Hence, for all these economies, the equilibrium is (at least) $\mathrm{CP}$ optimal. ${ }^{6}$ This result is established in Proposition 1 and Corollary 1. If there is no-trade at the PO equilibrium allocation of $(\bar{\omega}, \bar{u})$, we are only able to provide a weaker result: each open neighborhood of $(\bar{\omega}, \bar{u})$ contains open subsets of economies with CP efficient equilibria (in view of Proposition 1). However, it also contains other, distinct, open sets of economies such that the FOCs are violated at the equilibrium, which is, then, $\mathrm{CP}$ suboptimal. To show this, for each economy with a no-trade, $\mathrm{PO}$ equilibrium and each open neighborhood of $(\bar{\omega}, \bar{u})$, we construct an open set of economies such that there is a direction of equilibrium price adjustment $\overrightarrow{d \vec{p}}$ satisfying

a. $\overrightarrow{d \tilde{p}}=D_{\tilde{b}} \tilde{p} \overrightarrow{d \vec{b}}$, for some feasible $\overrightarrow{d \vec{b}}$, i.e., $\overrightarrow{d \tilde{p}}$ is attainable for some feasible portfolio perturbation,

$$
\text { b. } \overrightarrow{d \widetilde{p}} \Lambda\left(\bar{\lambda}^{n}, \bar{z}\right)>>0 \text {. }
$$

Hence, the equilibria of these economies cannot be $\mathrm{CP}$ efficient, because the equilibrium price adjustment induced by $\overrightarrow{d \vec{b}}$ entails a Pareto improvement upon the equilibrium allocation. This final result is reported in Proposition 2. Notice that this property is essentially independent of $\mathrm{PO}$ of the equilibrium of the reference economy, while it rests heavily on the absence of trade at the equilibrium (see also [5]).

We can now proceed to fill in the details, following the road map just outlined. Our analysis rests on several standard generic properties of equilibria, summarized in Lemma 1.

Let $\mathcal{E}_{N T}^{P O}$ be the set of economies with a Pareto optimal initial endowment.

Lemma 1 There is a relatively open and dense subset of $\mathcal{E}_{N T}^{P O}, \mathcal{E}_{N T}^{P O R}$, such that, for each $(\bar{\omega}, \bar{u}) \in \mathcal{E}_{N T}^{P O R}$, there is an open set $B_{\varepsilon}(\bar{\omega}, \bar{u}) \subset \mathcal{E}$ such that, for each $(\omega, u) \in B_{\varepsilon}(\bar{\omega}, \bar{u})$, a. the equilibrium is regular, unique and $D_{p} \tilde{Z}$ has full rank $(S+1)(C-1)$ at $\tilde{b}=\bar{b}(\bar{p}, \bar{q})$,

\footnotetext{
6 "At least", because the open set necessarily includes also economies with a PO equilibrium.
} 
b. if $(H-1) J \geq(S+1)(C-1)$, at the equilibrium $(\bar{p}(\omega, u), \bar{q}(\omega, u))$ of each $(\omega, u) \in B_{\varepsilon}(\bar{\omega}, \bar{u})$, the matrix

$$
D_{\tilde{b}} \tilde{Z}^{*} \equiv\left[\ldots,\left[D_{\tilde{b}_{h}} \tilde{z}_{h}\left(\bar{p}, \bar{q} ; \tilde{b}_{h}\right)-D_{\tilde{b}_{H}} \tilde{z}_{H}\left(\bar{p}, \bar{q} ; \tilde{b}_{H}\right)\right]^{T}, \ldots\right]
$$

has full row rank at $\tilde{b}=\bar{b}(\bar{p}, \bar{q})$.

Proof The properties in (a) are always satisfied for economies in some open neighborhood of an economy in $\mathcal{E}_{N T}^{P O R}$. (b) is essentially established in [2] and, exactly in this form, in [5].

Notice that (b) in Lemma 1 rules out, among others, economies with identical, homothetic preferences.

We now establish our preliminary results. The first is that, if an economy has a PO equilibrium, then, for all the economies sufficiently close, the equilibrium allocation may be Pareto dominated only by the $\tilde{b}$-conditional equilibria associated with portfolios arbitrarily close to the equilibrium one. This follows immediately from the definition of Pareto optimality.

In the sequel, $(\bar{p}(\omega, u), \bar{q}(\omega, u))$ is the vector of equilibrium prices of the economy $(\omega, u)$, while $\tilde{p}(\tilde{b} ; \omega, u)$ is the vector of $\tilde{b}$-conditional equilibrium prices given $(\omega, u)$ and some feasible $\tilde{b}$.

Lemma 2 Let $(\bar{\omega}, \bar{u}) \in \mathcal{E}_{N T}^{P O R}$. Then, for each $\varepsilon>0$, $\varepsilon$ sufficiently small, there is $\xi(\varepsilon)>0$ such that, for the equilibrium of each $(\omega, u) \in B_{\varepsilon}(\bar{\omega}, \bar{u}) \subset \mathcal{E}$, there is no Pareto superior, $\tilde{b}$-conditional equilibrium with $\|\tilde{b}-\bar{b}(\bar{p}(),. \bar{q}(.) ; \bar{\omega}, \bar{u})\|>$ $\xi(\varepsilon)$. Moreover, for each sequence $\left\{\varepsilon^{v}\right\}_{v=1}^{v=\infty}, \varepsilon^{v} \rightarrow 0$, each associated sequence $\left\{\xi\left(\varepsilon^{v}\right)\right\}_{v=1}^{v=\infty}$ satisfies $\xi\left(\varepsilon^{v}\right) \rightarrow 0$.

Proof The first part of the lemma is obvious, because the matrix of asset payoffs, $R$, has full rank and the consumption set is bounded below.

To show the second part, we proceed by contradiction. Suppose that there is a sequence $\left\{\varepsilon^{v}\right\}_{v=1}^{v=\infty}, \varepsilon^{v} \rightarrow 0$, such that some associated sequence $\left\{\xi\left(\varepsilon^{v}\right)\right\}_{v=1}^{v=\infty}$ is bounded away from 0 , i.e., such that $\xi\left(\varepsilon^{v}\right) \rightarrow \bar{\xi}>0$. Then, we can construct a sequence $\left\{\left(\omega^{v}, u^{v}\right)\right\}_{v=1}^{\infty}$, with $\left(\omega^{v}, u^{v}\right) \in B_{\varepsilon^{v}}(\bar{\omega}, \bar{u})$, for each $v$, satisfying two properties: first, $\left(\omega^{v}, u^{v}\right) \rightarrow(\bar{\omega}, \bar{u})$; secondly, for each $v$, there is a portfolio profile $\tilde{b}^{v}$ such that its associated $\tilde{b}^{v}$-conditional equilibrium allocation $\tilde{x}\left(\tilde{b}^{v} ; \omega^{v}, u^{v}\right)$ Pareto dominates the actual equilibrium allocation $x\left(\omega^{v}, u^{v}\right)$, while $\left\|\tilde{b}^{v}-\bar{b}\left(\bar{p}(),. \bar{q}(.) ; \omega^{v}, u^{v}\right)\right\| \geq \bar{\xi}$. Since all these sequences can be taken to be convergent, $\tilde{b}^{v} \rightarrow b^{\circ},\left(q\left(\omega^{v}, u^{v}\right), \tilde{p}\left(\tilde{b}^{v} ; \omega^{v}, u^{v}\right)\right) \rightarrow\left(q^{\circ}, \tilde{p}^{\circ}\right)$, and $\tilde{x}^{v} \rightarrow x^{\circ}$. Moreover, by continuity,

$$
\tilde{V}_{h}\left(\tilde{p}^{\circ}, \bar{q} ; \tilde{b}_{h} ; \bar{\omega}, \bar{u}\right) \geq V_{h}(\bar{p}, \bar{q} ; \bar{\omega}, \bar{u}) .
$$

Since, for each $v,\left\|\tilde{b}^{v}-\bar{b}\left(\bar{p}(),. \bar{q}(.) ; \omega^{v}, u^{v}\right)\right\| \geq \bar{\xi},\left\|\tilde{b}^{0}-\bar{b}(\bar{p}(),. \bar{q}(.) ; \bar{\omega}, \bar{u})\right\| \geq$ $\bar{\xi}>0$. Given that $R$ has full rank, this implies $x^{\circ} \neq \bar{x}$. This is impossible because utility functions are strictly-concave: since $x^{\circ} \neq \bar{x}$, for each $\pi \in$ $[0,1], x^{\pi}=\pi \bar{\omega}+(1-\pi) x^{\circ}$ is a feasible allocation strictly Pareto superior to $\bar{x}$. This contradicts the PO of $\bar{x}$. 
This result actually holds for all the economies sufficiently close to one with a regular, PO equilibrium: absence of trade plays no role in its proof.

By the previous Lemma, for economies close to any $(\bar{\omega}, \bar{u}) \in \mathcal{E}_{N T}^{P O R}$, each possible portfolio with a Pareto superior associated allocation must lie in some bounded neighborhood of the actual equilibrium portfolio $\bar{b}(\bar{\omega}, \bar{u})$. For economies in $\mathcal{E}_{N T}^{P O R}$, this means that $\|\tilde{b}\| \leq \xi$, for some $\xi>0$. Hence, without any loss of generality, we can restrict the analysis to feasible portfolios lying in the $\xi$-ball, for some $\xi>0$. Let

$$
\mathbf{S}_{\xi} \equiv\{\tilde{b} \mid\|\tilde{b}\| \leq \xi\} \cap\left\{\tilde{b} \mid \sum_{h} \tilde{b}_{h}=0\right\} \subset \mathbf{R}^{H J} .
$$

Given $\bar{\mu} \in \mathbb{R}_{++}^{H}$, define the map $\tilde{T}_{\bar{\mu}}(\tilde{b} ; \omega, u), \tilde{T}_{\bar{\mu}}: \mathbf{S}_{\xi} \times \mathcal{E} \longrightarrow \mathbb{R}$,

$$
\tilde{T}_{\bar{\mu}}(\tilde{b} ; \omega, u) \equiv \sum_{h} \bar{\mu}_{h} \tilde{V}_{h}\left(\tilde{p}(\tilde{b} ; \omega, u), \bar{q}(\omega, u), \tilde{b}_{h} ; \omega, u\right),
$$

so that $\tilde{T}_{\bar{\mu}}(\tilde{b} ; \omega, u)$ incorporates both the direct and the indirect effects of a portfolio reallocation on the - weighted - sum of the indirect utilities. Bear in mind that we are considering just economies with an initial endowment close to be PO, so that we can assume that their equilibria and $\tilde{b}$-conditional equilibria are unique, at least for $\tilde{b}$ sufficiently close to the equilibrium ones. ${ }^{7}$ Also, bear in mind that our analysis is local and refers to economies satisfying the properties of Lemma 1 . Hence, $\tilde{T}_{\bar{\mu}}(\tilde{b} ; \omega, u)$ is a well-defined, smooth function, because $(\tilde{p}(\tilde{b} ; \omega, u), \bar{q}(\omega, u))$ is a regular equilibrium and shares these properties. Given $\bar{\mu} \in \mathbb{R}_{++}^{H}$, define the optimization problem: choose

$$
\widetilde{b} \in \arg \max \tilde{T}_{\bar{\mu}}(\widetilde{b} ; \bar{\omega}, \bar{u}) \text { subject to } \widetilde{b} \in \mathbf{S}_{\xi} .
$$

Lemma 3 shows that problem (2) provides a partial characterization of $\mathrm{CP}$ optimal allocations.

Lemma 3 Let $(\bar{\omega}, \bar{u})$ be any economy with an equilibrium $(\bar{p}, \bar{q})$. If the associated portfolio $\bar{b}$ solves optimization problem (2) for some $\mu>>0$, then the equilibrium is CP efficient.

Proof Otherwise, there would be some $\tilde{b} \in \mathbf{S}_{\xi}$ such that, at the associated $\tilde{b}$-conditional equilibrium $\tilde{p}(\tilde{b} ; \omega, u)$,

$$
\tilde{V}_{h}\left(\tilde{p}(\tilde{b} ; \bar{\omega}, \bar{u}), \bar{q}(\bar{\omega}, \bar{u}), \tilde{b}_{h} ; \bar{\omega}_{h}, \bar{u}_{h}\right) \geq V_{h}\left(\bar{p}(\bar{\omega}, \bar{u}), \bar{q}(\bar{\omega}, \bar{u}) ; \bar{\omega}_{h}, \bar{u}_{h}\right),
$$

for each $h$ and with some strict inequality. Then, $\tilde{T}_{\mu}(\tilde{b} ; \bar{\omega}, \bar{u})>\tilde{T}_{\mu}(\bar{b} ; \bar{\omega}, \bar{u})$, for $\bar{b}=\bar{b}(\bar{p}(\bar{\omega}, \bar{u}), \bar{q}(\bar{\omega}, \bar{u}) ; \bar{\omega}, \bar{u})$, because $\mu>>0$.

\footnotetext{
7 Technically, we are considering only economies in the (open) connected component of regular economies with a unique equilibrium and a unique $\tilde{b}$-conditional equilibrium.
} 
The key step in our proof is to observe that, for all $(\omega, u)$ in some open neighborhood of $(\bar{\omega}, \bar{u}) \in \mathcal{E}_{N T}^{P O R}, \tilde{T}_{\mu}(\tilde{b} ; \omega, u)$ is a strictly-concave function of $\tilde{b}$, for each $\mu$ in some open set $M \subset \mathbb{R}_{++}^{H}$, when restricted to feasible portfolio perturbations and to their induced price adjustments. This implies that the optimal solutions to problem (2) can be characterized in terms of its first order conditions (FOCs). This result is purely local, meaning that strictconcavity of $\tilde{T}_{\mu}(\tilde{b} ; \omega, u)$ holds for small perturbations of the equilibrium portfolios. However, in view of Lemma 2, these are the only perturbations which matter, for our purposes.

Lemma 4 Let $(\bar{\omega}, \bar{u}) \in \mathcal{E}_{N T}^{P O R}$. Then, there is an open set $B_{\delta}(\bar{\omega}, \bar{u}) \subset \mathcal{E}$ such that, at each $(\omega, u) \in B_{\delta}(\bar{\omega}, \bar{u})$ and at each $\mu$ in an open set $M \subset \mathbb{R}_{++}^{H}$, the map $\tilde{T}_{\mu}(\widetilde{b} ; \omega, u)$ is strictly-concave.

Proof Let $\tilde{Z}($.$) be the \tilde{b}$-conditional aggregate excess demand map. Fix $\bar{\mu}=\left[\ldots, \frac{1}{\lambda_{h}^{0}}, \ldots\right]$. Define the map

$$
T_{\bar{\mu}}(p, \tilde{b}) \equiv \sum_{h} \bar{\mu}_{h} \widetilde{V}_{h}\left(p, \bar{q}(\omega, u) ; \tilde{b}_{h}\right)
$$

where $p$ is now an arbitrary price vector. The simplest way to establish the Lemma is to consider the derivative of $T_{\bar{\mu}}(p, \tilde{b})$ restricted to the set of $\tilde{b}$-conditional equilibrium spot prices. In fact, we are going to show that, locally, $D_{(p, \tilde{b})}^{2} T_{\bar{\mu}}(p, \tilde{b})$ is negative-definite when restricted to the direction $\left[D_{\tilde{b}} \tilde{p} \overrightarrow{d \tilde{b}}, \overrightarrow{d \tilde{b}}\right]$, where $\overrightarrow{d \tilde{b}}$ is any feasible portfolio perturbation, i.e., one with $\sum_{h} d \tilde{b}_{h}=0$, while, by the implicit function theorem,

$$
D_{\tilde{b}} \tilde{p}=-\left[D_{p} \tilde{Z}\right]^{-1} D_{\tilde{b}} \tilde{Z}
$$

so that

$$
\nabla_{\tilde{b}} \tilde{T}_{\bar{\mu}}(\tilde{b} ; \omega, u)=\nabla_{p} T_{\bar{\mu}}(p, \tilde{b})\left[-\left[D_{p} \tilde{Z}\right]^{-1} D_{\tilde{b}} \tilde{Z}\right]+\nabla_{\tilde{b}} T_{\bar{\mu}}(p, \tilde{b})
$$

The unrestricted derivative of $T_{\bar{\mu}}(p, \tilde{b})$ with respect to portfolios and commodity prices is given by

$$
\begin{aligned}
\nabla_{(p, \widetilde{b})} T_{\bar{\mu}}(p, \widetilde{b}) & =\left[\left[\ldots, \sum_{h} \mu_{h} \frac{\partial \widetilde{V}_{h}}{\partial p^{s c}}, \ldots\right],\left[\ldots, \mu_{h} \nabla_{\widetilde{b}_{h}} \widetilde{V}_{h}, \ldots\right]\right] \\
& =\left[\left[\ldots,-\sum_{h} \mu_{h} \widetilde{\lambda}_{h}^{s} \widetilde{z}_{h}^{s c}, \ldots\right],\left[\ldots, \mu_{h} \nabla_{\widetilde{b}_{h}} \widetilde{V}_{h}, \ldots\right]\right] .
\end{aligned}
$$


Hence, by direct computation, at the equilibrium, $D_{(p, \tilde{b})}^{2} T_{\bar{\mu}}(p, \widetilde{b})$ is

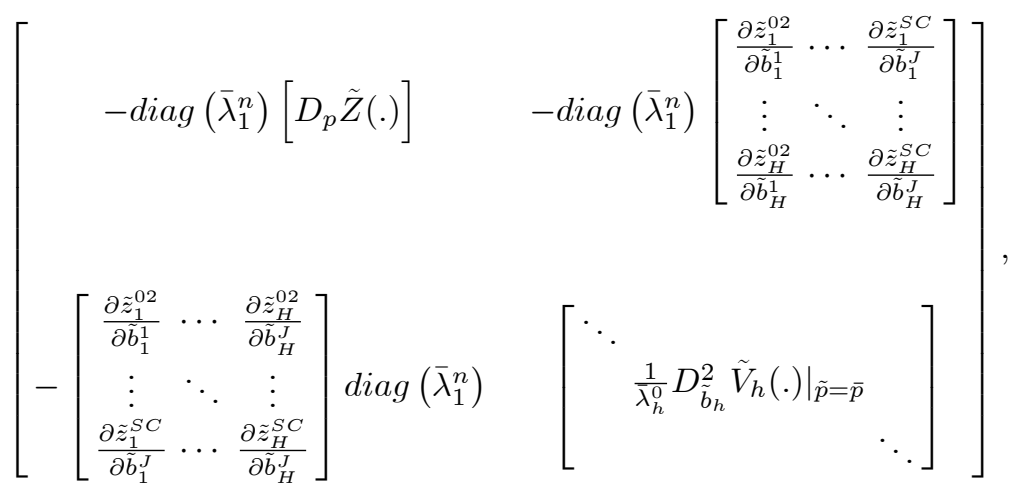

where $\operatorname{diag}\left(\bar{\lambda}_{1}^{n}\right)$ is the diagonal, $((S+1)(C-1))$-dimensional matrix with non-zero coefficients given by the vector of agent 1's equilibrium Lagrange multipliers, normalized by $\bar{\lambda}_{1}^{0}$.

This simple structure of $D_{(p, \tilde{b})}^{2} T_{\bar{\mu}}(p, \tilde{b})$ follows from two special features of no-trade, Pareto optimal allocations: first, by our choice of the vector $\bar{\mu}$, at the equilibrium

$$
\nabla_{p} T_{\bar{\mu}}(p, \tilde{b})=-\sum_{h} \bar{\mu}_{h} \operatorname{diag}\left(\bar{\lambda}_{h}\right) \bar{z}_{h}=-\operatorname{diag}\left(\bar{\lambda}_{1}^{n}\right) \sum_{h} \bar{z}_{h}=0,
$$

because, by PO, the vectors $\bar{\lambda}_{h}^{n}$ are $h$-invariant. Second, we can ignore all the terms $\frac{\partial \bar{\lambda}_{h}^{s n}}{\partial \tilde{b}_{h}^{s}} \bar{z}_{h}^{s c}$, because the equilibrium allocation is no-trade, so that $\bar{z}_{h}=0$, for each $h$. Hence, the sub-matrices have the simple structure reported above. By construction,

$$
\begin{aligned}
& \overrightarrow{d \vec{b}}\left[D_{\tilde{b}}^{2} \tilde{T}_{\bar{\mu}}\right] \overrightarrow{d \tilde{b}^{T}} \\
& \equiv\left[-\overrightarrow{d \tilde{b}}\left[D_{p} \tilde{Z}\right]^{-1} D_{\tilde{b}} \tilde{Z}^{T} \overrightarrow{d \tilde{b}}\right]\left[D_{(p, \tilde{b})}^{2} T_{\bar{\mu}}\right]\left[\begin{array}{c}
-\left[D_{p} \tilde{Z}\right]^{-1} D_{\tilde{b} \tilde{Z}^{T}} \overrightarrow{d \tilde{b}}^{T} \\
\overrightarrow{d \tilde{b}}^{T}
\end{array}\right] \\
& =\operatorname{diag}\left(\bar{\lambda}_{1}^{n}\right) \overrightarrow{d \vec{b}}\left[D_{\tilde{b}} \tilde{Z}^{T}\right]\left[D_{p} \tilde{Z}\right]^{-1}\left[D_{\tilde{b}} \tilde{Z}^{T}\right] \overrightarrow{d \tilde{b}^{T}}+\sum_{h} \frac{1}{\lambda_{h}^{\sigma}} \overrightarrow{d \tilde{b}} D_{\tilde{b}_{h}}^{2} \tilde{V}_{h} \overrightarrow{d \tilde{b}}^{T} \leq 0,
\end{aligned}
$$

with strict inequality for each $\neq 0$. Indirect utility functions are strictlyconcave, by assumption. Since there are no income effects, $D_{p} \tilde{Z}$ is negativedefinite at each no-trade equilibrium. Thus, its inverse is also negativedefinite. Hence, the quadratic form is negative-definite so that the last inequality follows immediately. ${ }^{8}$ Hence, strict-concavity of $\tilde{T}_{\bar{\mu}}(\tilde{b})$ holds at

${ }^{8}$ The first term of the sum is negative-definite at the equilibria of economies in the connected component of $(\bar{\omega}, \bar{u})$. The second term is always such. To obtain negative-definiteness of $D_{\tilde{b}}^{2} \tilde{T}_{\bar{\mu}}$ in the relevant directions, we also need that the values of the excess demand vectors are suitably small. 
$\left((\bar{\omega}, \bar{u}), \bar{\mu}=\left[\ldots, \frac{1}{\lambda_{h}^{0}}, \ldots\right]\right)$. Define the map

$N(d \tilde{b} ; \omega, u) \equiv \operatorname{diag}\left(\bar{\lambda}_{1}^{n}\right)\left[\overrightarrow{d \vec{b}} D_{\tilde{b}} \tilde{Z}^{T}\right]\left[D_{p} \tilde{Z}\right]^{-1} D_{\tilde{b}} \tilde{Z} \overrightarrow{d \vec{b}} \vec{b}^{T}+\sum_{h} \frac{1}{\bar{\lambda}_{h}^{0}} \overrightarrow{d \vec{b}} D_{\tilde{b}_{h}}^{2} \tilde{V}_{h} \overrightarrow{\widetilde{d}}^{T}$

and, without any loss of generality, just consider $\widetilde{b} \in \mathbf{S}_{\xi}$. Given any $(\omega, u)$ with equilibrium portfolio $\bar{b}(\omega, u)$, let $\widetilde{d b}(\omega, u) \equiv[\widetilde{b}-\bar{b}(\omega, u)]$ and restrict the analysis to $\widetilde{b} \in \mathbf{S}_{\xi}$. In view of the previous Lemma and for our purposes, this implies no loss of generality, if we consider only economies in some sufficiently small open neighborhood of $(\bar{\omega}, \bar{u})$. We conclude the argument claiming that, for some sufficiently small, open $B_{\delta}(\bar{\omega}, \bar{u})$, for each $(\omega, u) \in B_{\delta}(\bar{\omega}, \bar{u}), N(\widetilde{d b} ; \omega, u)<0$ for each $\widetilde{d b} \neq 0$, which means that $\tilde{T}_{\bar{\mu}}($. is strictly-concave, locally. Otherwise, since $\mathbf{S}_{\xi}$ is compact, we can construct a sequence $\left\{\widetilde{d b}^{v},\left(\omega^{v}, u^{v}\right)\right\}_{v=0}^{v=\infty}$ such that $\left(\omega^{v}, u^{v}\right) \rightarrow(\bar{\omega}, \bar{u}), \widetilde{d b}^{v} \rightarrow{\widetilde{d b^{*}} \text { and }}^{v}$ $N\left(\widetilde{d b^{v}} ; \omega^{v}, u^{v}\right) \geq 0$ for each $v$. Since $N($.$) is continuous, N\left(\widetilde{d b^{*}} ; \bar{\omega}, \bar{u}\right) \geq 0$. This is impossible, because $N\left(\widetilde{d b^{*}} ; \bar{\omega}, \bar{u}\right)<0$ for each $\widetilde{d b} \in \mathbf{S}_{\xi}$. Given that strict-concavity is an open property, the same result holds for each $\mu$ in some open $M \subset \mathbb{R}_{++}^{H}$.

Consider now only economies in $B_{\delta}(\bar{\omega}, \bar{u})$. Given that problem $(2)$ is strictly-concave, its optimal solution is characterized by its first order conditions

$$
\operatorname{FOC}_{\bar{\mu}}(\tilde{b}) \equiv\left[\begin{array}{c}
\vdots \\
\left.\sum_{h} \bar{\mu}_{h} \nabla_{p} \tilde{V}_{h}\right|_{\tilde{b}_{h}} \nabla_{\tilde{b}_{h}} \tilde{p}^{T}-\phi+\left.\bar{\mu}_{h} \nabla_{\tilde{b}_{h}} \tilde{V}_{h}\right|_{\tilde{p}} \\
\vdots \\
\sum_{h} \tilde{b}_{h}
\end{array}\right]=0
$$

with Lagrange multiplier $\phi$. Bear in mind that $\operatorname{FOC}_{\bar{\mu}}(\tilde{b})$ takes into account the adjustment of the $\tilde{b}$-conditional equilibrium prices.

At an equilibrium, $\left.\nabla_{\tilde{b}_{h}} \tilde{V}_{h}\right|_{\tilde{p}}=0$, for each $h$. Hence, getting rid of the multiplier $\phi$, we can rewrite $F O C_{\bar{\mu}}(\tilde{b})$ in compact form as

$$
\operatorname{FOC}_{\bar{\mu}}^{*}(\tilde{b}) \equiv \quad\left[\begin{array}{c}
\vdots \\
\left.\sum_{h} \bar{\mu}_{h} \nabla_{p} \tilde{V}_{h}\right|_{\tilde{b}_{h}}\left[\nabla_{\tilde{b}_{h}} \tilde{p}-\nabla_{\tilde{b}_{H}} \tilde{p}\right]^{T} \\
\vdots
\end{array}\right]=0,
$$

$$
F O C_{\bar{\mu}}^{*}(\tilde{b})=\quad\left[\ldots, \bar{\mu}_{h} \bar{\lambda}_{h}^{0}, \ldots\right] \Lambda\left(\bar{\lambda}^{n}, \bar{z}\right)^{T}\left[-D_{p} \tilde{Z}\right]^{-1} D_{\tilde{b}} \tilde{Z}^{*}=0 .
$$


Under our assumptions, and because of Lemma $3,\left[\left[-D_{p} \tilde{Z}\right]^{-1} D_{\tilde{b}} \tilde{Z}^{*}\right]$ has maximal row rank $(S+1)(C-1)$. Hence, $F O C_{\bar{\mu}}^{*}(\tilde{b})=0$ if and only if

$$
\left[\ldots, \bar{\mu}_{h} \bar{\lambda}_{h}^{0}, \ldots\right] \Lambda\left(\bar{\lambda}^{n}, \bar{z}\right)^{T}=0
$$

is satisfied for $\bar{\mu}=\left[\ldots, \frac{1}{\lambda_{h}^{0}}, \ldots\right]$. Evidently, at a PO equilibrium allocation, [1] $\Lambda\left(\bar{\lambda}^{n}, \bar{z}\right)^{T}=0$, because of market-clearing and equality of the normalized Lagrange multipliers of the individual optimization problems. In Proposition 7, we construct an open set of economies with CP efficient equilibria: they are any open neighborhood of an economy with a PO equilibrium allocation, a suitably small amount of trade, and a matrix $\Lambda\left(\bar{\lambda}^{n}, \bar{z}\right)$ with full row rank.

Proposition 1 Let $(\bar{\omega}, \bar{u}) \in \mathcal{E}_{N T}^{P O R}$. Then, there is an open set $B_{\delta}(\bar{\omega}, \bar{u}) \subset \mathcal{E}$ such that:

a) there is an economy $\left(\omega^{\circ}, u^{\circ}\right) \in B_{\delta}(\bar{\omega}, \bar{u})$ with a PO equilibrium such that $\Lambda\left(\lambda^{n^{\circ}}, z^{\circ}\right)$ has maximal row rank $(S+1)(C-1)$,

b) for each economy $\left(\omega^{\circ}, u^{\circ}\right) \in B_{\delta}(\bar{\omega}, \bar{u})$ satisfying a), there is an open neighborhood $B_{\varphi}\left(\omega^{\circ}, u^{\circ}\right) \subset B_{\delta}(\bar{\omega}, \bar{u})$ such that each $(\omega, u) \in B_{\varphi}(\bar{\omega}, \bar{u})$ has a unique, $C P$ efficient equilibrium.

Proof Given $(\bar{\omega}, \bar{u})$ and the associated open set $B_{\delta}(\bar{\omega}, \bar{u})$, with the properties discussed in Lemma 4 , by a standard argument, i.e., modulo an appropriate perturbation of $(\omega, u)$, there is $\left(\omega^{\circ}, u^{\circ}\right) \in B_{\delta}(\bar{\omega}, \bar{u})$ satisfying $\left.a\right)$.

At the equilibrium of $\left(\omega^{\circ}, u^{\circ}\right), \Lambda\left(\lambda^{n^{\circ}}, z^{\circ}\right)[1]^{T}=0$. By regularity of the equilibrium, and maximal row rank of $\Lambda\left(\lambda^{n^{\circ}}, z^{\circ}\right)$, there is an open neighborhood $B_{\varphi}\left(\omega^{\circ}, u^{\circ}\right) \subset B_{\delta}(\bar{\omega}, \bar{u})$ such that, at the equilibrium of each $(\omega, u) \in$ $B_{\varphi}\left(\omega^{\circ}, u^{\circ}\right), \Lambda\left(\lambda^{n}, z\right)[\mu]^{T}=0$ has a strictly positive solution $\mu(\omega, u)$, which can be made arbitrarily close to [1] by choosing $\varphi$ sufficiently small. Pick the open neighborhood $B_{\varphi}(\bar{\omega}, \bar{u})$. By construction, for each $(\omega, u) \in B_{\varphi}(\bar{\omega}, \bar{u})$, optimization problem (2) is strictly-concave given $\mu(\omega, u)$ sufficiently close to $\left[\ldots, \frac{1}{\lambda_{h}^{0}(\omega, u)}, \ldots\right]$, since $(\omega, u) \in B_{\varphi}\left(\omega^{\circ}, u^{\circ}\right) \subset B_{\delta}(\bar{\omega}, \bar{u})$. Moreover, $\bar{b}(\omega, u)$ solves its FOCs. Hence, the unique equilibrium of $(\omega, u)$ is CP efficient.

The structure of the proof should have made clear why CP efficiency may fail in a neighborhood of economies with a PO equilibrium allocation, but with arbitrary amount of trade. When $\|\bar{z}\|$ is "large", we loose the simple structure of the Hessian matrix $D_{(p, \tilde{b})}^{2} T_{\bar{\mu}}$, described in Lemma 4, because the derivatives of the Lagrange multipliers with respect to $(p, \tilde{b})$ matter. Moreover, we may loose negative-definiteness of the matrix $\left[D_{p} \tilde{Z}\right]$. Lemma 2 still holds, so that we can restrict the analysis to portfolios close to the equilibrium ones, but this is not enough to establish CP efficiency.

Remark 1 Our construction of this open set of economies imposes no special restrictions on the utility functions. All we need is that a PO no-trade equilibrium is regular and unique. This is always true. The proof of Proposition 
1 then rests just on the maximal row rank of $\Lambda\left(\lambda^{n^{\circ}}, z^{\circ}\right)$, and on a suitably small level of trade. When $(C-1)(S+1)>H$, both properties hold at each equilibrium of economies close to the original one, modulo some small, appropriate endowment-utility perturbation.

The main implication of Proposition 1 is immediate and stated as a Corollary: given an economy with a PO equilibrium, and a sufficiently small level of trade, the equilibria of all the neighboring economies are $\mathrm{CP}$ efficient

Corollary 1 Let $(\widehat{\omega}, \widehat{u}) \in \mathcal{E}^{P O R}$. If the equilibrium level of trade is sufficiently small and $\operatorname{rank} \Lambda(\widehat{\lambda}, \widehat{z})=(S+1)(C-1)$, then there is an open set $B_{\varphi}(\widehat{\omega}, \widehat{u}) \subset \mathcal{E}$ such that, for each $(\omega, u) \in B_{\varphi}(\widehat{\omega}, \widehat{u})$, the associated equilibrium is $C P$ efficient.

The next proposition establishes that, in each $B_{\delta}(\bar{\omega}, \bar{u}) \in \mathcal{E}$ with $(\bar{\omega}, \bar{u}) \in$ $\mathcal{E}_{N T}^{P O R}$, and for $\delta$ sufficiently small, there is an open set $B_{\delta}^{C P I} \subset B_{\delta}(\bar{\omega}, \bar{u})$ where $\mathrm{CP}$ inefficiency holds. We could establish this result showing that there is an open set of economies whose equilibria necessarily violate the necessary conditions that a CP efficient $\tilde{b}$-conditional equilibrium must satisfy. However, it is simpler to proceed directly, showing that, under the maintained assumptions, for some open set $B_{\delta}^{C P I}$, at each equilibrium, $\overrightarrow{d \vec{p}} \Lambda\left(\tilde{\lambda}^{n}, \tilde{z}\right)>>0$ for some price adjustment vector $\overrightarrow{d \tilde{p}}$ attainable as a $\tilde{b}$-conditional equilibrium for some feasible portfolio perturbation. This immediately implies that the equilibrium is CP inefficient. In view of Stiemke's Lemma, the two arguments of proof are equivalent.

Proposition 2 Let $(\bar{\omega}, \bar{u}) \in \mathcal{E}_{N T}^{P O R}$. Then, for each open set $B_{\delta}(\bar{\omega}, \bar{u}) \subset \mathcal{E}$ with $\delta \leq \bar{\delta}$, for some $\bar{\delta}>0$, there is an open set $B_{\delta}^{C P I} \subset B_{\delta}(\bar{\omega}, \bar{u})$, such that, for each $(\omega, u) \in B_{\delta}^{C P I}$, the unique equilibrium is CP inefficient.

The argument of the proof is similar to the one used to establish Proposition 11, in [5]. For completeness, we sketch it in Appendix.

Remark 2 Consider an economy with $H \leq(S+1)(C-1)$ agents. Pick a PO equilibrium. Evidently, the results of Lemma 2 and 3 still hold, because they do not depend upon the number of agents and commodities. On the other hand, there are no open sets of economies with CP efficient equilibria. The reason is that, at the economy with a PO equilibrium, $\Lambda\left(\bar{\lambda}^{n}, \bar{z}\right)\left[\ldots, \mu_{h} \lambda_{h}^{0}, \ldots\right]^{T}=0$ for $\mu=\left[\ldots, \frac{1}{\lambda_{h}^{0}}, \ldots\right]$. However, since $H \leq(S+1)(C-1), \Lambda(\lambda, z)$ has maximal rank $H$ at each equilibrium of a generic set of economies. Hence, at the equilibrium of a generic economy, there is no solution but $\phi=0$ to $\Lambda\left(\lambda^{n}, z\right) \phi^{T}=0$. Hence, by Stiemke's Lemma, there are directions $\overrightarrow{d \tilde{p}}$ such that $\nabla_{p} \tilde{V}_{h} \overrightarrow{d \vec{p}}^{T}>0$, for each $h$, and equilibria are, in fact, typically $\mathrm{CP}$ inefficient.

We conclude presenting an example. We pick $(\bar{\omega}, \bar{u}) \in \mathcal{E}_{N T}^{P O R}$. We first show that $\tilde{T}_{\bar{\mu}}(\widetilde{b} ; \omega, u)$ is strictly-concave at $\bar{\mu}=\frac{1}{\lambda^{0}}$. Hence, by Lemma 4 , 
the first order conditions for a maximum of $\tilde{T}_{\bar{\mu}}(\widetilde{b} ; \omega, u)$ are necessary and sufficient for each economy sufficiently close to $(\bar{\omega}, \bar{u})$.

Next, picking economies with appropriate matrices $\Lambda\left(\lambda^{n}, z\right)$, we provide:

i. an open neighborhood of an economy with a PO equilibrium where all the equilibria are CP efficient (as in Corollary 1),

ii.a an open set of economies, arbitrarily close to $(\bar{\omega}, \bar{u})$ with a unique $\mathrm{CP}$ efficient equilibrium (since the set is open, for most economies the equilibrium is $\mathrm{CP}$ efficient, but not PO), (as in Proposition 1)

ii.b an open set of economies such that the equilibrium is CP inefficient (as in Proposition 2).

Example 1 The reference economy is Cobb-Douglas, with two spots in the second period and just one asset, inside money. There are 4 agents, with utility functions

$$
u_{h}\left(x_{h}\right)=\sum_{s=0}^{s=2} \beta_{h}^{s}\left(\gamma_{h}^{s} \ln x_{h}^{s 1}+\left(1-\gamma_{h}^{s}\right) \ln x_{h}^{s 2}\right)
$$

The discount factors are $\beta_{1}=\left(1,\left(\frac{1}{2}-\theta\right),\left(\frac{1}{2}+\theta\right)\right), \beta_{2}=\beta_{3}=\left(1, \frac{1}{2}, \frac{1}{2}\right)$ and $\beta_{4}=\left(1,\left(\frac{1}{2}+\theta\right),\left(\frac{1}{2}-\theta\right)\right)$. The other parameters are described in Table 1 .

$\left[\begin{array}{cccccccccc}\multicolumn{7}{c}{\text { Table 1: Parameters of the economies }} \\ & \omega_{h}^{01} & \omega_{h}^{02} & \omega_{h}^{11} & \omega_{h}^{12} & \omega_{h}^{21} & \omega_{h}^{22} & \gamma_{h}^{01} & \gamma_{h}^{11} & \gamma_{h}^{21} \\ h=1 & 4-a_{1}^{01} & 4+a_{1}^{01} & 6+a_{1}^{11} & 2-a_{1}^{11} & 4 & 4 & \frac{1}{2} & \frac{3}{4} & \frac{1}{2} \\ h=2 & 9 & 3 & 6-a_{2}^{11} & 6+a_{2}^{11} & 6 & 6 & \frac{3}{4} & \frac{1}{2} & \frac{1}{2} \\ h=3 & 2 & 6 & 6 & 2 & 2-a_{3}^{21} & 6+a_{3}^{21} & \frac{1}{4} & \frac{3}{4} & \frac{1}{4} \\ h=4 & 6+a_{1}^{01} & 6-a_{1}^{01} & 6-a_{1}^{11}+a_{2}^{11} & 6+a_{1}^{11}-a_{2}^{11} & 9+a_{3}^{21} & 3-a_{3}^{21} & \frac{1}{2} & \frac{1}{2} & \frac{3}{4}\end{array}\right]$

The $\tilde{b}$-conditional, indirect utility function of agent $h$ can be written as

$$
\begin{aligned}
\tilde{V}_{h}(.)= & \ln \left(\tilde{p}^{0} \omega_{h}^{0}-\widetilde{b}_{h}\right)+\beta_{h}^{1} \ln \left(\tilde{p}^{1} \omega_{h}^{1}+\widetilde{b}_{h}\right)+\beta_{h}^{2} \ln \left(\tilde{p}^{2} \omega_{h}^{2}+\widetilde{b}_{h}\right) \\
& -\left(\left(1-\gamma_{h}^{0}\right) \ln p^{0}+\beta_{h}^{1}\left(1-\gamma_{h}^{1}\right) \ln p^{1}+\beta_{h}^{2}\left(1-\gamma_{h}^{2}\right) \ln p^{2}\right) .
\end{aligned}
$$

The first result, (i.), does not require us to be very specific about the perturbation of the parameters. For the second and third, we will set $a_{1}^{01}=\theta$, and $a_{2}^{11}=a_{3}^{21}=\theta^{2}$. Equilibria are CP efficient if $a_{1}^{11}=0, \mathrm{CP}$ inefficient if $a_{1}^{11}=2 \theta$.

First, notice that, for each $\theta,(\bar{p}, \bar{q})=(1,1,1,1)$ is the equilibrium and there 
is no asset trade. Moreover, when $\theta=0$, the endowment is PO and the equilibrium is no-trade.

By direct computation, the $\tilde{b}$-conditional spot equilibrium prices (computed for feasible portfolios, i.e., setting $\tilde{b}_{4}=-\sum_{h=1}^{3} \tilde{b}_{h}$ ) are

$$
\tilde{p}(\tilde{b})=\left(1+\frac{\tilde{b}_{2}-\tilde{b}_{3}}{35}, \quad 1-\frac{b_{1}+b_{3}}{36-a_{1}^{11}}, \quad 1+\frac{\tilde{b}_{1}+\tilde{b}_{2}+2 \tilde{b}_{3}}{35-2 \theta^{2}}\right) .
$$

First, let us check that, in a neighborhood of the reference no-trade and $\mathrm{PO}$ economy, we can describe CP efficient allocations as a solution to the strictly-concave optimization problem (2).

Fix $\bar{\mu}=\left[\ldots, \frac{1}{\lambda_{h}^{0}}, \ldots\right]$. The easiest way to check for the strict-concavity of optimization problem (2) is to compute the second order derivative of $T_{\bar{\mu}}(p, \tilde{b})$ with respect to $(p, \tilde{b})$ and then check the sign of the quadratic form for the feasible directions, i.e., the ones with $\sum_{h} \tilde{b}_{h}=0$, and the associated $\tilde{b}$-conditional equilibrium prices. By direct computation, using the formulas obtained in the proof of Lemma 4 , at the reference no-trade and $\mathrm{PO}$ equilibrium,

$$
D_{(p, \tilde{b})}^{2} T_{\bar{\mu}}=\left[\begin{array}{ccccccc}
\frac{35}{4} & 0 & 0 & \frac{1}{2} & \frac{1}{4} & \frac{3}{4} & \frac{1}{2} \\
0 & \frac{9}{2} & 0 & -\frac{1}{8} & -\frac{1}{4} & -\frac{1}{8} & -\frac{1}{4} \\
0 & 0 & \frac{35}{8} & -\frac{1}{4} & -\frac{1}{4} & -\frac{3}{8} & -\frac{1}{8} \\
\frac{1}{2} & -\frac{1}{8} & -\frac{1}{4} & -\frac{1}{4} & 0 & 0 & 0 \\
\frac{1}{4} & -\frac{1}{4} & -\frac{1}{4} & 0 & -\frac{1}{6} & 0 & 0 \\
\frac{3}{4} & -\frac{1}{8} & -\frac{3}{8} & 0 & 0 & -\frac{1}{4} & 0 \\
\frac{1}{2} & -\frac{1}{4} & -\frac{1}{8} & 0 & 0 & 0 & -\frac{1}{6}
\end{array}\right] .
$$

To compute the quadratic form on the subspace of the feasible perturbations, we restrict its computation to the directions $\overrightarrow{\tilde{d}} \equiv\left[-D_{\tilde{p}} \tilde{Z}^{-1} D_{\tilde{b}} \tilde{Z} \overrightarrow{d \vec{b}}, \overrightarrow{d \tilde{b}},-\sum_{h<H} d \tilde{b}_{h},\right]$, where $\overrightarrow{d \vec{b}} \equiv\left[d \tilde{b}_{1}, \ldots, d \tilde{b}_{H-1}\right]$, i.e., we pre-multiply $D_{(p, \tilde{b})}^{2} T_{\bar{\mu}}$ by $\vec{d}$

$$
\left[\begin{array}{ccccccc}
0 & -\frac{1}{36} & \frac{1}{35} & 1 & 0 & 0 & -1 \\
\frac{1}{35} & 0 & \frac{1}{35} & 0 & 1 & 0 & -1 \\
-\frac{1}{35} & -\frac{1}{36} & \frac{2}{35} & 0 & 0 & 1 & -1
\end{array}\right]
$$

and post-multiply it by $\vec{d}^{T}$. We obtain

$$
\left[D_{\tilde{b}}^{2} \tilde{T}_{\bar{\mu}}\right]^{T}=\frac{1}{10080}\left[\begin{array}{l}
-4271-1716-1787 \\
-1716-3468-1680 \\
-1787-1680-4451
\end{array}\right] .
$$

Its leading minors have signs $[-,+,-]$. Hence, it is negative-definite and the first order conditions of (2) are sufficient for CP efficiency. Replace the feasibility constraint into the objective function. Then, the FOCs are given by

$$
\left[\left.\bar{\mu}_{h} \nabla_{\widetilde{b}_{h}} \widetilde{V}_{h}\right|_{\bar{p}}-\left.\bar{\mu}_{H} \nabla_{\widetilde{b}_{H}} \widetilde{V}_{H}\right|_{\bar{p}}\right]+\left[\sum_{h} \bar{\mu}_{h} \nabla_{p} \widetilde{V}_{h}\right] \nabla_{\widetilde{b}_{h}} \widetilde{p}=0 \text {, for each } h<H .
$$


Given that $\left.\nabla_{\widetilde{b}_{h}} \widetilde{V}_{h}\right|_{\bar{p}}=0$ at each equilibrium, if $\left[\sum_{h} \bar{\mu}_{h} \nabla_{p} \widetilde{V}_{h}\right]=0$, the equilibrium must be $\mathrm{CP}$ efficient.

Let us now consider the matrix $\Lambda\left(\bar{\lambda}^{n}, \bar{z}\right)$. When evaluated at the equilibrium of the reference economy, it is given by

$$
\Lambda\left(\bar{\lambda}^{n}, \bar{z}\right)=\left[\begin{array}{cccc}
-\bar{\lambda}_{1}^{s n} \bar{z}_{1}^{s 2} & -\bar{\lambda}_{2}^{s n} \bar{z}_{2}^{s 2} & -\bar{\lambda}_{3}^{s n} \bar{z}_{3}^{s 2} & -\bar{\lambda}_{4}^{s n} \bar{z}_{4}^{s 2} \\
-\bar{z}_{1}^{02} & -\bar{z}_{2}^{02} & -\bar{z}_{3}^{02} & -\bar{z}_{4}^{02} \\
-\left(\frac{1}{2}-\theta\right) \bar{z}_{1}^{12} & -\frac{1}{2} \bar{z}_{2}^{12} & -\frac{1}{2} \bar{z}_{3}^{12} & -\left(\frac{1}{2}+\theta\right) \bar{z}_{4}^{12} \\
0 & -\frac{1}{2} \bar{z}_{2}^{22} & -\frac{1}{2} \bar{z}_{3}^{22} & -\left(\frac{1}{2}-\theta\right) \bar{z}_{4}^{22}
\end{array}\right]
$$

and it is trivial at each no-trade equilibrium. We consider three cases:

$i$. Pick any perturbation of the initial endowments such that the associated matrix $\Lambda\left(\bar{\lambda}^{n}, \bar{z}\right)$ has full rank. Clearly, $\Lambda\left(\bar{\lambda}^{n}, \bar{z}\right)[1]^{T}=0$ and the equilibrium is PO. By continuity, at the equilibrium of each economy in some open neighborhood of $(\bar{\omega}, \bar{u}), \Lambda\left(\lambda^{n}, z\right)[\mu]^{T}=0$ for some $\mu>>$. Therefore, the equilibrium is $\mathrm{CP}$ efficient for an open set of economies.

ii.a Fixing the values of the $a_{h}^{s c}$ as explained above, we obtain

$$
\Lambda\left(\bar{\lambda}^{n}, \bar{z}\right)=\left[\begin{array}{cccc}
-\bar{\lambda}_{1}^{s n} \bar{z}_{1}^{s 2} & -\bar{\lambda}_{2}^{s n} \bar{z}_{2}^{s 2}-\bar{\lambda}_{3}^{s n} \bar{z}_{3}^{s 2} & -\bar{\lambda}_{4}^{s n} \bar{z}_{4}^{s 2} \\
\theta & 0 & 0 & -\theta \\
-\left(\frac{1}{2}-\theta\right) a_{1}^{11} & \frac{1}{2} \theta^{2} & 0 & \left(\frac{1}{2}+\theta\right)\left(a_{1}^{11}-\theta^{2}\right) \\
0 & 0 & \frac{1}{2} \theta^{2} & -\left(\frac{1}{2}-\theta\right) \theta^{2}
\end{array}\right]
$$

When $a_{1}^{11}=0$, evidently, $\Lambda\left(\bar{\lambda}^{n}, \widehat{z}\right) \mu^{T}=0$ has a strictly positive solution, $\widehat{\mu}=(1,(1+2 \theta),(1-2 \theta), 1)$. Hence, the equilibrium satisfies the FOCs of (2): the allocation is CP efficient. Since $\Lambda\left(\bar{\lambda}^{n}, \widehat{z}\right)$ has maximal rank, by continuity, this property is open.

ii.b Set $a_{1}^{11}=2 \theta$. We can write each solution to $\Lambda\left(\bar{\lambda}^{n}, \bar{z}\right) \mu^{T}=0$ as $\widehat{\mu}_{1}=\widehat{\mu}_{4}=1$ and $\widehat{\mu}_{3}=\left(1-2 \theta^{2}\right)$. However, $\widehat{\mu}_{2}=2 \theta-7<0$. By Stiemke Lemma, there is a direction $\overrightarrow{d \widetilde{p}}$ such that $\overrightarrow{d \vec{p}} \Lambda\left(\bar{\lambda}^{n}, \bar{z}\right)>>0$. For instance, $\psi[1,1,1] \Lambda\left(\bar{\lambda}^{n}, \bar{z}\right)=\left[2, \frac{1}{2}, \frac{1}{2}, 1\right] \theta^{2} \psi>>0$. To compute the change in portfolios inducing such a price variation, we solve $\overrightarrow{d \vec{b}}=\left[D_{\widetilde{b}} \widetilde{p}\right]^{-1} \overrightarrow{d \vec{p}}^{T}$ at 
$\overrightarrow{d \vec{p}}=[1,1,1]$, i.e,

$$
\left[\begin{array}{c}
\widetilde{d b}_{1} \\
\widetilde{d b_{2}} \\
\widetilde{d b}_{3}
\end{array}\right]=\left[\begin{array}{ccc}
\frac{35}{2} & 3 \theta-54 \theta^{2}-\frac{35}{2} \\
\frac{35}{2} & 18-\theta & \frac{35}{2}-\theta^{2} \\
-\frac{35}{2} & 18-\theta & \frac{35}{2}-\theta^{2}
\end{array}\right]\left[\begin{array}{l}
1 \\
1 \\
1
\end{array}\right] \psi=\left[\begin{array}{c}
\left(\theta^{2}+3 \theta-54\right) \\
\left(-\theta^{2}-\theta+53\right) \\
\left(-\theta^{2}-\theta+18\right)
\end{array}\right] \psi=\overrightarrow{\overrightarrow{d b}}(\psi) .
$$

By construction, the $\widetilde{b}$-conditional equilibrium price vector associated with the (feasible) portfolio $\left(\overrightarrow{\widetilde{d b}}(\psi),\left(\theta^{2}-\theta-17\right) \psi\right)$ is $\widetilde{p}(\overrightarrow{\widetilde{d b}}(\psi))=[(1+\psi),(1+\psi),(1+\psi)]$.

We now compute the values of $\widetilde{V}_{h}($.$) , for each h$, replacing the $\widetilde{b}$-conditional spot prices with the map $\widetilde{p}(\overrightarrow{d \vec{b}}(\psi))$ and using the portfolio perturbation $\overrightarrow{\overrightarrow{d b}}(\psi)$. By direct computation, the derivatives of $\widetilde{V}_{h}(),. h=1, . ., 4$, with respect to $\psi$, evaluated at $\psi=0$, are given by the strictly positive vector $\left[\frac{1}{4}, \frac{1}{24}, \frac{1}{16}, \frac{1}{12}\right] \theta^{2}$. Hence, the equilibrium is CP inefficient.

In Figure 1a and 1b, we present the changes of $\widetilde{V}_{h}($.$) , for h=1,2,3,4$, in a neighborhood of $\psi=0$, for $\theta=\frac{1}{20}$ and $\theta=\frac{1}{100} .{ }^{9}$

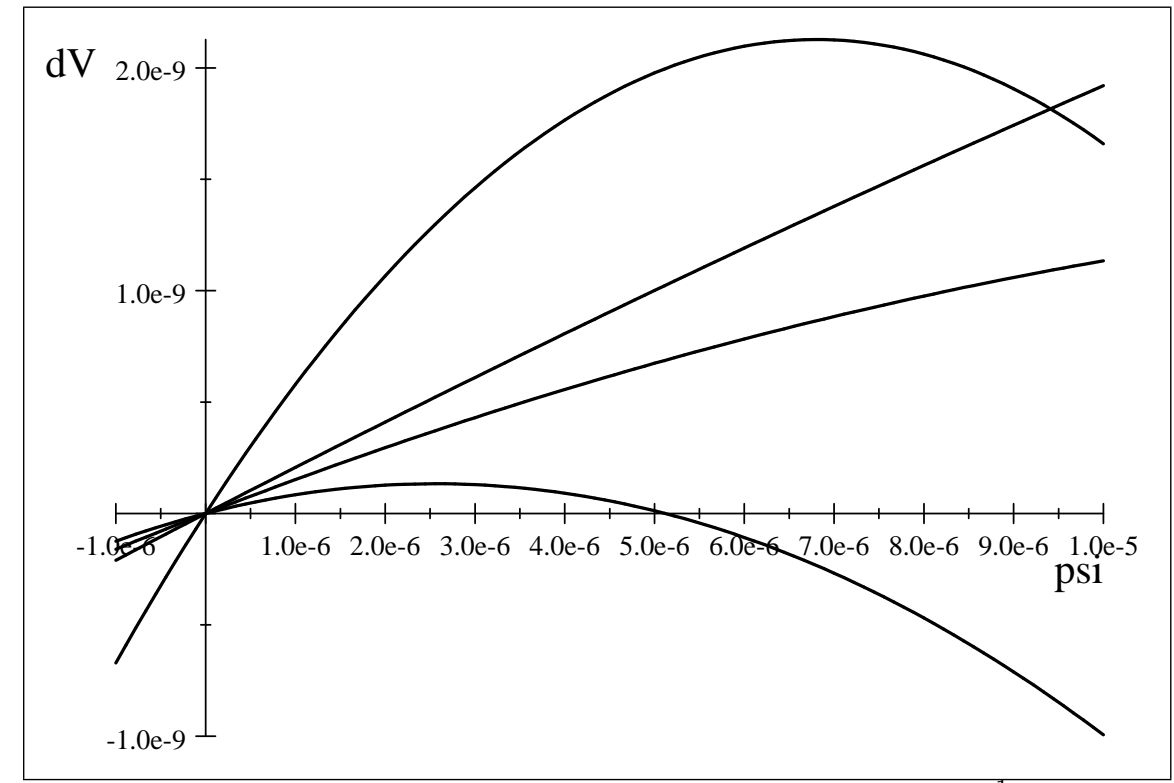

Figure 1a: Changes in the equilibrium utilities, at $\theta=\frac{1}{20}$

${ }^{9}$ The values of $\widetilde{V}_{h}($.$) are scaled so that their values are 0$ at $\psi=0$ and that all the graphs are readable in the same figure. 


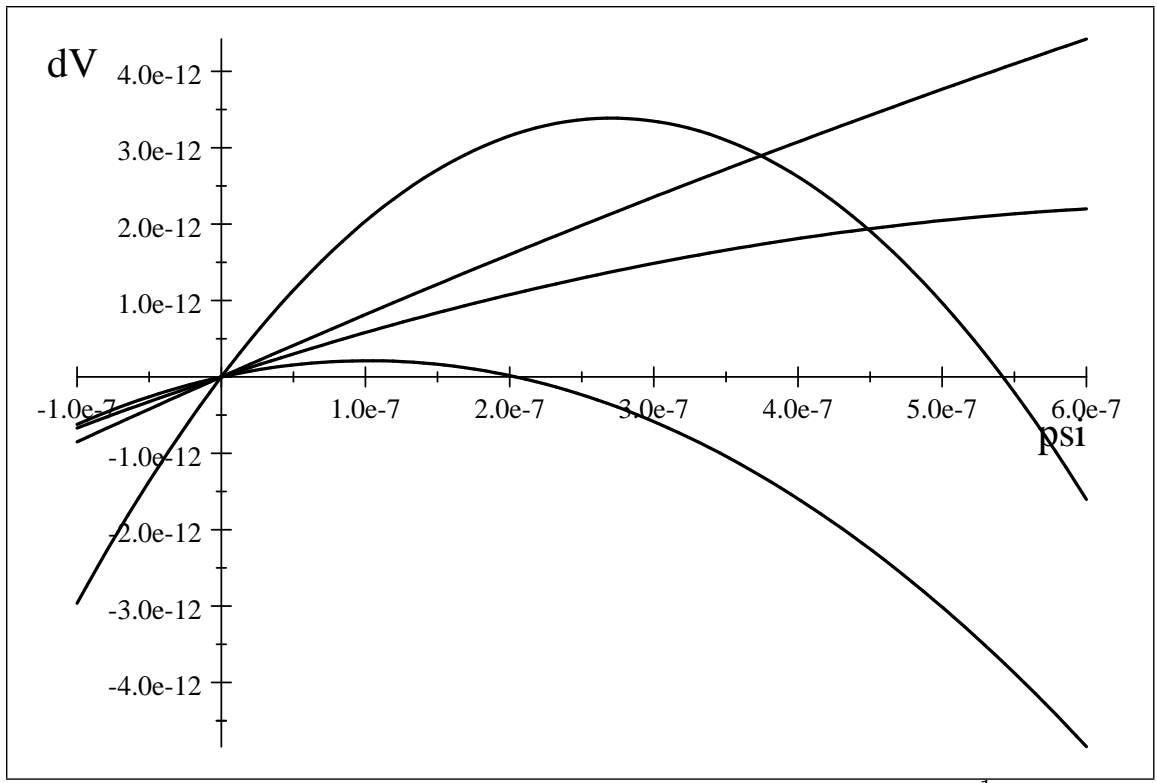

Figure 1b: Changes in the equilibrium utilities, at $\theta=\frac{1}{100}$

The last two parametric examples define two distinct paths, parameterized by $\theta$, in the space of the economies. For all the ones on the path defined in (ii.a), and all the ones sufficiently close to them, the unique equilibrium is CP efficient. For all the economies close to the path defined in (ii.b), the unique equilibrium is CP inefficient. The two paths cross at the economy with the PO, no-trade equilibrium. In terms of efficiency properties, this economy is critical. In fact, it is a critical point of the map $D(.) \equiv \sum_{k} d_{k}^{2}$, where $k$ indexes the collection of the determinants $d_{k}$ of all the sub-matrices of $\Lambda\left(\lambda^{n}, z\right)$.

\section{Conclusions}

In this paper, we have analyzed constrained efficiency of equilibria in GEI with a finitely large number of agents for economies close to one with a Pareto optimal equilibrium allocation. Suppose that, at this equilibrium, the matrix describing the impact of price changes on the attainable utilities has maximal rank equal to the number of non-numeraire commodities. Then, for all the economies sufficiently close, the equilibrium is constrained efficient, at least with respect to small portfolio perturbations. It is globally constrained optimal if the equilibrium volume of trade at the initial equilibrium is sufficiently small. To the contrary suppose that, at the equilibrium, there is no-trade. Then, each open neighborhood of the reference economy contains open sets of economies with a unique constrained optimal equilibrium and other open sets of economies with a unique, constrained inefficient 
equilibrium. We formally establish this result for economies where there is no trade at the equilibrium (so that the matrix has rank 0), but it may hold whenever the row rank of the matrix is not full.

\section{Appendix}

Proof of Proposition 2. Pick $(\bar{\omega}, \bar{u}) \in \mathcal{E}_{N T}^{P O R}$ and restrict the analysis to economies in the open set considered in Lemma 1. Hence, for each economy in any open set in $B_{\varepsilon}(\bar{\omega}, \bar{u})$ there is a unique and regular equilibrium. We split the proof into two steps.

Step 1: Given $(\bar{\omega}, \bar{u})$, for each open set $B_{\delta}(\bar{\omega}, \bar{u})$ there is $\left(\bar{\omega}, u^{\prime}\right) \in$ $B_{\delta}(\bar{\omega}, \bar{u})$, the set of Lemma 1 , with a unique no-trade equilibrium satisfying

1. the normalized vectors of Lagrange multipliers satisfy $\frac{\bar{\lambda}_{1}^{1}}{\bar{\lambda}_{1}^{0}}>\ldots>\frac{\bar{\lambda}_{H}^{1}}{\bar{\lambda}_{H}^{0}}$,

2. the matrix $D_{\tilde{b}} \tilde{Z}^{*} \equiv\left[\ldots,\left[D_{\tilde{b}_{h}} \tilde{z}_{h}\left(\bar{p}, \bar{q} ; \tilde{b}_{h}\right)-D_{\tilde{b}_{1}} \tilde{z}_{1}\left(\bar{p}, \bar{q} ; \tilde{b}_{1}\right)\right]^{T}, \ldots\right]$ has

full row rank at $\tilde{b}_{h}=b_{h}(\bar{p}, \bar{q})$, for each $h$,

3. prices satisfy $\left(\bar{p}\left(\bar{\omega}, u^{\prime}\right), \bar{q}\left(\bar{\omega}, u^{\prime}\right)\right)=(\bar{p}(\bar{\omega}, \bar{u}), \bar{q}(\bar{\omega}, \bar{u}))$.

Proof. (1) can be established exploiting a standard, locally linear, perturbation of the utility functions and relabeling agents, (2) is a generic property of equilibria and, by construction, is satisfied at $(\bar{\omega}, \bar{u})$ (remember Lemma 1). Both properties can be established using utility perturbations with no effect on equilibrium prices and allocation. We can construct an economy $\left(\bar{\omega}, u^{\prime}\right)$ satisfying (1-2) using perturbations of the utility functions which do not affect equilibrium prices. Hence, (3) holds, too.

Step 2: $\quad$ Pick any $\left(\bar{\omega}, u^{\prime}\right)$ satisfying (1-3) above, and consider any open neighborhood $B_{\eta}\left(\bar{\omega}, u^{\prime}\right) \subset B_{\delta}(\bar{\omega}, \bar{u})$. Then, there is some open set $B_{\delta}^{C P I} \subset$ $B_{\eta}\left(\bar{\omega}, u^{\prime}\right)$ such that, for each $(\omega, u) \in B_{\delta}^{C P I}$, the unique, regular equilibrium is $\mathrm{CP}$ inefficient.

Proof. First, observe that, for feasible portfolios,

$$
D_{\tilde{b}} \tilde{p}=-\left[D_{p} \tilde{Z}\right]^{-1}\left[D_{\tilde{b}} \tilde{Z}^{*}\right] .
$$

Since $\left[D_{p} \tilde{Z}\right]^{-1}$ has full rank $(S+1)(C-1)$, while $\left[D_{\tilde{b}} \tilde{Z}^{*}\right]$ has maximal row rank $(S+1)(C-1), D_{\tilde{b}} \tilde{p}$ has full row rank. Hence, $\tilde{p}(\tilde{b})$, locally, is onto and our proof reduces to establish conditions under which there is a solution to $\overrightarrow{d \vec{p}} \Lambda\left(\lambda^{n}(\omega, u), z(\omega, u)\right)>>0$ for each $(\omega, u) \in B_{\delta}^{C P I}$, an open subset of $B_{\eta}\left(\bar{\omega}, u^{\prime}\right)$.

By construction, at the equilibrium, $\left(\bar{p}\left(\bar{\omega}, u^{\prime}\right), \bar{q}\left(\bar{\omega}, u^{\prime}\right)\right)=(\bar{p}(\bar{\omega}, \bar{u}), \bar{q}(\bar{\omega}, \bar{u}))$ and the consumption and portfolio allocation is also the same. Fix $u^{\prime}$ and 
pick an endowment vector $\widehat{\omega}$ close to $\bar{\omega}$, and such that $\left(\bar{p}\left(\widehat{\omega}, u^{\prime}\right), \bar{q}\left(\widehat{\omega}, u^{\prime}\right)\right)=$ $\left(\bar{p}\left(\bar{\omega}, u^{\prime}\right), \bar{q}\left(\bar{\omega}, u^{\prime}\right)\right)$, while the associated matrix $\overrightarrow{d \tilde{p}} \Lambda\left(\widehat{\lambda}^{n}, \widehat{z}\right)$ is given by

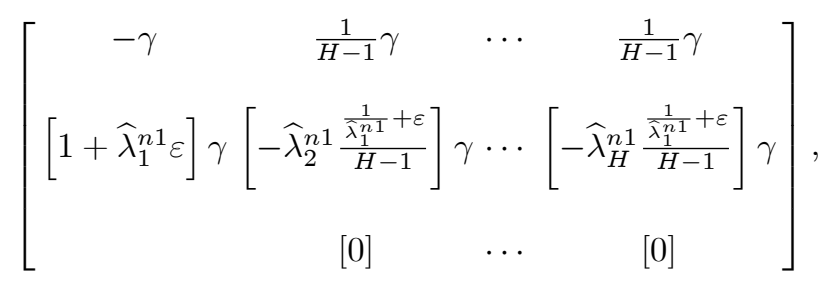

with non-zero excess demand just for commodity 2 at $s=0,1$.

Evidently, for each $\gamma>0$ and $0<\varepsilon<\frac{1}{\hat{\lambda}_{2}^{n 1}}-\frac{1}{\hat{\lambda}_{1}^{n 1}}$,

$[\ldots, 1, \ldots] \Lambda\left(\widehat{\lambda}^{n}, z^{\prime}\right)=\left[\widehat{\lambda}_{1}^{n 1} \varepsilon, \frac{1-\frac{\widehat{\lambda}_{2}^{n 1}}{\hat{\lambda}_{1}^{n 1}}-\widehat{\lambda}_{2}^{n 1} \varepsilon}{H-1}, \ldots, \frac{1-\frac{\widehat{\lambda}_{H}^{n 1}}{\hat{\lambda} \widehat{\lambda}_{1}^{n 1}}-\widehat{\lambda}_{H}^{n 1} \varepsilon}{H-1}\right] \gamma>>0$.

As argued, the result of the proposition follows immediately, since $\Lambda\left(\lambda^{n}, z\right)$, evaluated at the equilibrium, is continuous in $(\omega, u)$.

By regularity, if such a $\left(\widehat{\omega}, u^{\prime}\right)$ exists, there is $B_{\delta}^{C P I} \subset B_{\eta}\left(\bar{\omega}, u^{\prime}\right) \subset B_{\delta}(\bar{\omega}, \bar{u})$ such that, for each $(\omega, u) \in B_{\delta}^{C P I}$, the equilibrium is $\mathrm{CP}$ inefficient, as required. Hence, to conclude, we just need to show that there is some $\left(\widehat{\omega}, u^{\prime}\right)$ with the stated properties. This can be shown using a standard perturbation of the initial endowment, or following verbatim the proof of Proposition 11 in [5].

\section{References}

1. Citanna, A., Kajii, A., Villanacci, A., 1998. Constrained Suboptimality in Incomplete Markets: A General Approach and Two Applications. Economic Theory $11,495-521$.

2. Geanakoplos, J., Polemarchakis, H.M., 1986. Existence, Regularity and Constrained Suboptimality of Competitive Allocations When the Asset Market Is Incomplete. In W.P. Heller, R.M. Starr and D. Starrett (eds.), Uncertainty, Information and Communication: Essays in Honor of K.J. Arrow, Vol. III. Cambridge University Press, Cambridge, 65-95.

3. Kajii, A., 1992. Constrained Suboptimality with Many Agents. CARESS Working Paper \#92-29, University of Pennsylvania.

4. Mas-Colell, A., 1987. On the Theorem of Geanakoplos-Polemarchakis. Unpublished manuscript, Harvard University.

5. Mendolicchio, C., Pietra, T., 2019. A Re-examination of Constrained Pareto Inefficiency in Economies with Incomplete Markets. Journal of Mathematical Economics 80, 39-55.

6. Stiglitz, J.E., 1982. The Inefficiency of the Stock Market Equilibrium. Review of Economic Studies 49, 241-261. 